\title{
Secular evolution in young galaxies
}

\section{Bruce G. Elmegreen}

IBM T.J. Watson Research Center, 1101 Kitchawan Road, Yorktown Heights, NY 10598 USA email: bge@us.ibm.com

\begin{abstract}
Young galaxies viewed at high redshift have high turbulent velocities, high star formation rates, high gas fractions, and chaotic structures, suggesting wild instabilities during which giant gas clumps form and make stars in their dense regions, stir other disk stars and gas, and transport angular momentum outward with a resulting net mass flow inward (e.g., Ceverino et al. 2010). At $z=1.5,40 \%$ of star-forming galaxies have significant clumps (Elmegreen et al. 2007; Wuyts et al. 2012), and in these, $10 \%-20 \%$ of the stellar mass is in clumps that last $\sim 150$ Myr (Elmegreen et al. 2009; Wuyts et al. 2012). The thick disk and bulge in modern galaxies could form in this phase. The similarity in the $\alpha / \mathrm{Fe}$ ratio (Meléndez et al. 2008), K-giant abundances (Bensby et al. 2010) and ages for the Milky Way bulge and thick disk suggest they formed at the same time. High dispersion gas at $z \sim 1.5$ can do this because it makes the young disk thick and the SF clumps big enough to drive fast secular evolution (Elmegreen et al. 2006; Genzel et al. 2008; Bournaud et al. 2009). Local analogues might be present in dynamically young galaxies like BCDs (Elmegreen et al. 2012). The high fraction of $z \sim 1.5$ galaxies with massive clumps suggests clump formation is a long-lived phase and that clump torques should last $\sim 1$ Gyr or more even if individual clumps come and go on shorter timescales. Clump formation may cease when stars finally dominate the disk mass (Cacciato et al. 2012).
\end{abstract}

Keywords. galaxies: bulges, galaxies: dwarf, galaxies: evolution, galaxies: high-redshift

\section{References}

Bensby, T., Alves-Brito, A., Oey, M. S., Yong, D., \& Meléndez, J. 2010, A $\& A$ 516, L13

Bournaud, F., Elmegreen, B. G., \& Martig, M. 2009, ApJ 707, L1

Cacciato, M., Dekel, A., \& Genel, S. MNRAS 421, 818

Ceverino, D., Dekel, A., \& Bournaud, F. 2010, MNRAS 404, 2151

Elmegreen, B. G. \& Elmegreen, D. M. 2006, ApJ 650, 644

Elmegreen, D. M., Elmegreen, B. G., Ravindranath, S., \& Coe, D. A. 2007, ApJ 658, 763

Elmegreen, B. G., Elmegreen, D. M., Fernandez, M. X., \& Lemonias, J. J. 2009, ApJ 692, 12

Elmegreen, B. G., Zhang, H.-X., \& Hunter, D. A. 2012, ApJ 747, 105

Genzel, R. et al. 2008, ApJ 687, 59

Meléndez, et al. 2008, A\&A 484, L21

Wuyts, S., et al. 2012, ApJ 753, 114 